\title{
DESCRIPTION DE LA FEMELLE DE TRIATOMA MAXIMA (UHLER 1894)
}

\author{
Par F. LARROUSSE
}

Grâce à l'obligeance de M. le professeur Bouvier qui a bien voulu mettre à notre disposition l'importante collection d'Hémiptères du Muséum national d'histoire naturelle de Paris, nous avons eu la bonne fortune de trouver dans un carton de Réduvidés non classés, une forme intéressante du genre Triatoma. Cette magnifique espèce, dans un parfait état de conservation nous a de suite frappé par sa très grande taille : $4 \mathrm{~cm}$. 4. Aucune des espèces signalées à ce jour ne présente de semblables dimensions. Toutefois Uhler, en 1894, avait décrit sous le nom de Conorhinus maximus un exemplaire $\sigma$ provenant de la basse Californie, dont la taille déjà remarquable était de $3 \mathrm{~cm}$. 5 . La description donnée par Uhler, que nous reproduisons dans son texte, peut s'appliquer en tous points à notre exemplaire, en faisant la seule remarque que Uhler a décrit un mâle, ce qui explique la dimension plus réduite 'qu'il signale.

\section{Conorhinus maximus, n. sp. $\sigma^{x}$.}

P.-R. Uhler. Proceedings of the California Acad. of. Sci., $2^{\mathrm{e}}$ série, vol. IV, I, p. 286.

Coal black, shining, narrower than $C$. dimidiatus Lat. Head much thicker than in any other species known to me, rough and transversely wrinkled, somewhat pubescent, the tip of tylus projecting over a notch, each side of which the cheek projects in a produced point, base of this cheek long triangular and scooped out; the buccular tip knoblike and protrated anteriorly; rostrum barely reaching upon the sternum; space behind the eye very short, coarsely wrinkled; antennæ mutilated. Pronotum moderately short, with a strongly constricted collum directly behind the head, the outer ends of which are drawn out and knob-like; anterior lobe very short and narrow, deeply sunken on the middle, with the tumid elevations each side set with sinuous series of coarse grains, the posterior lobe thick and wide, coarsely and unevenly wrinkled in several separate divisions, the divaricating lines almost obsolete; the lateral border thick, broadly curved, coarsely tuberculated below the slender waved carina, the humeral tubercle long, tumid, absorbing the lateral carina; posterior margin

Annales de Parastrologie, T, II, $\mathrm{N}^{\circ} 3,-$ Juillet 1924 , p. 207. 
almost straight and abruptly steep against the base of scutellum, each side of this obliquely curved. Scutellum coarsely knobbed at base, more finely towards the tip, deeply scooped out, the apical portion narrow, subcylindrical, ending in a knob-like tip. Corium minutely scabrous, and the clavus more coarsely so. Abdomen long, ovate, wider than the wingcovers, but not broadly expanded, with the margin brigth red all around; venter highly polished, transversely wrinkled.

Leng to tip of venter, $35 \mathrm{~mm}$. ; width of pronotum, $81 / 2 \mathrm{~mm}$.

Only one specimen, a male, has thus far been brought to my notice. It was kindly given to me by Dr. George H. Horn, as having been taken in Lower California. It differs from all species known to me by having the outer edge of the connexivum thickened, no sharp-edged, as in common to the large Mexican forms.

En résumé, le Triatoma maxima décrit par Uhler se caractérise par sa grande taille, sa coloration noire, brillante par place, et par son connexivum très épais bordé de rouge.

La présente description de la femelle concorde dans ses points principaux avec la description du mâle : nous sommes en droit, croyons-nous, d'identifier notre spécimen à Triatoma maxima. (Uhler, 1894).

Triatoma maxima (Uhler, 1894), $\sigma^{x}$; Larrousse, 1924, $q$.

\section{Femelle}

TAILlE. - Très grande espèce :

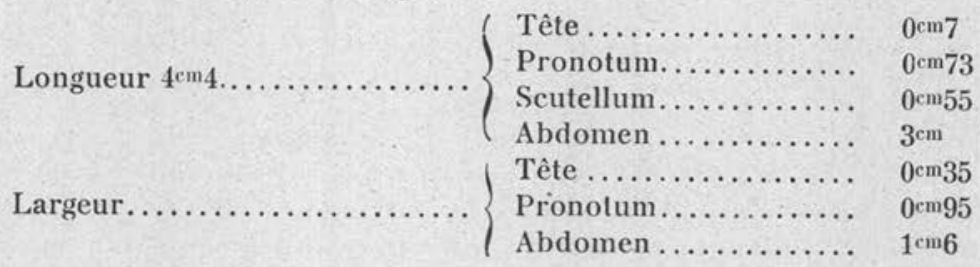

Coloration générale. - Noir brillant, principalement sur le pronotum et toute la face inférieure ; étroite bordure du connexivum rouge.

TÊTE. - Forte, large, rugueuse, la partie anté-oculaire 5 fois plus longue que la post-oculaire. Lobe médian proéminent, plus court que les lobes latéraux qui sont saillants et bien délimités sur leur bord supérieur par un sillon très net. Les antennes, longues, s'insèrent sur un tubercule court, dont la base est à égale distance du bord antérieur de l'œil et de l'apex de la tête ; $1^{\mathrm{er}}$ article atteignant à peine l'extrémité antérieure des lobes latéraux, $2^{\circ}$ article un peu 
plus de 3 fois $1 / 2$ plus long que le $1^{\text {er }}$, cet article est brun noir, couvert de courts poils mélangés à des poils plus longs et très rares, $3^{\circ}$ article sensiblement égal au $2^{\circ}$, recouvert d'un fin duvet jaune doré qui lui donne un aspect très clair, le $4^{e}$ article, la moitié du précédent, est également de couleur claire. Yeux très saillants, ocelles ne présentant rien de particulier. Rostre court, robuste, atteignant à peine le bord antérieur du thorax, $1^{\text {er }}$ article 3 fois plus court que le $2^{\text {e}}$, 3 un peu plus court que le $1^{\text {er }}$, brun et couvert de longs poils.

Thorax. - Pronotum sensiblement de la même longueur que la tête, lobe antérieur court, profondément divisé à sa partie médiane par un sillon profond, ce lobe est irrégulièrement et fortement granuleux et contraste avec le lobe postérieur qui est lisse et brillant; le lobe postérieur modérément dilaté, présente de chaque côté de la ligne médiane deux carènes qui sont obsolètes sur toute leur moitié postérieure, les angles latéraux sont peu saillants et délimités par une carène qui se perd insensiblement sur le lobe antérieur. Scutellum court, triangulaire, à bords

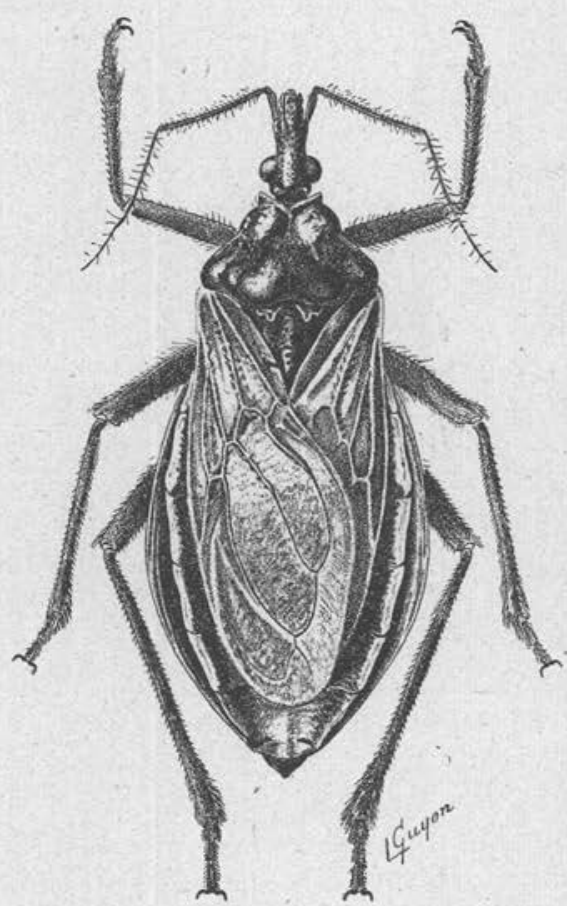

FIg. 1. - Trialoma maxima (Uhler), Q. $\times 1,64$. latéraux fortement sinueux, le disque est strié profondément, la pointe apicale est mousse.

Aвdomen. - Très allongé ; hémélytres courtes, laissant bien à découvert le connexivum et le dernier anneau; la coloration des hémélytres est uniformément sombre, cependant un peu plus claire sur les membranes qui ont une coloration brun de poix, plus foncée sur les nervures. Connexivum large, à bords très épais ; l'épaisseur du connexivum chez notre exemplaire à jeun est marquée par un double feuillet, le feuillet interne plus court est bordé par une étroite bande rouge qui s'atténue un peu au bord inférieur de chaque segment, le feuillet externe est plus large et de coloration 
plus claire que le reste du corps. Toute la face inférieure de l'abdomen est lisse, brillante, principalement sur le disque qui est déprimé, de fines stries parallèles sont visibles sur tous les segments.

Pattes. - Noires, l'extrémité des tibias qui sont dilatés et tous les tarses sont recouverts par des touffes de poils de couleur ocre ; les fémurs, également dilatés, présentent à leur apex un sillon sur
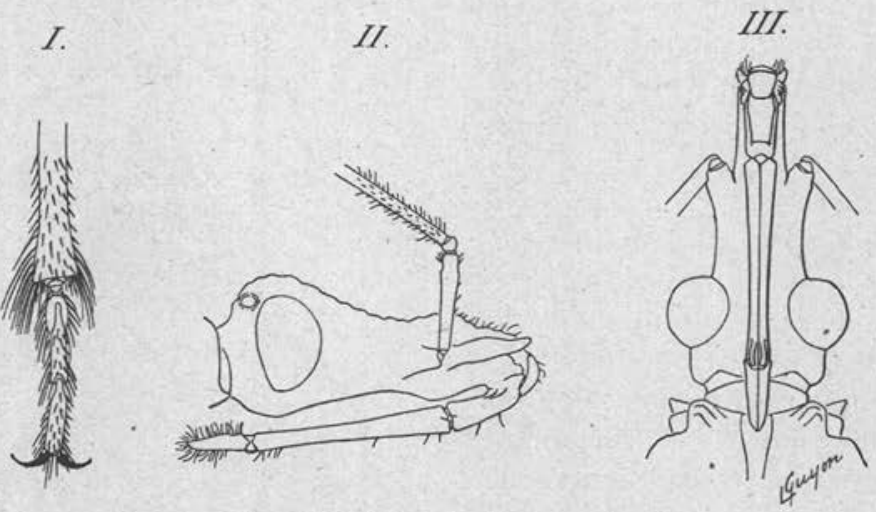

Fig. 2. - I, extrémité de la 1'e paire de pattes :

II, tête vue de profil ; III, tête vue en dessous. $\times 4,67$.

les bords internes et externes et deux crêtes formées de tubercules sur leur bord inférieur.

Cet exemplaire a été capturé au mois d'août 1914 par M. Diguet, sur le sommet d'un promontoire de $400 \mathrm{~m}$. d'altitude, connu sous le nom de Serro de las calaveras qui se trouve à proximité de la ville de la Pầz, Basse Californie, Mexique.

\section{RÉSUMÉ}

Dans cette courte note, nous décrivons la femelle de Triatoma maxima (Uhler, 1894). Ce Triatoma, qui est la plus grande espèce actuellement connue, est caractérisé par sa couleur noire brillante ; son connexivum très épais, bordé de rouge et par des touffes de poils couleur ocre à l'apex des tibias et sur les tarses. Les deux exemplaires actuellement connus proviennent de la basse Californie.

Laboratoire de Parasitologie de la Faculté de médecine de Paris. 